\section{Perceived and Actual Breast Cancer Risk}

\section{Optimistic and Pessimistic Biases}

\section{CELETTE SUGG SKINNER}

Mallinckrodt Institute of Radiology, Washington University School of Medicine, St Louis, Missouri, USA

\section{MATTHEW W. KREUTER}

Health Communications Research Laboratory, School of Public Health, Saint Louis University, St Louis, Missouri, USA

\section{SARAH KOBRIN}

Health Communications Research Laboratory, University of North Carolina School of Public Health, Chapel Hill, North Carolina, USA

\section{VICTOR J. STRECHER}

Director of Cancer Prevention and Control, University of Michigan Comprehensive Cancer Center, Ann Arbor, Michigan, USA

CELETTE SUGG SKINNER, PhD, is assistant professor at the Mallinckrodt Institute of Radiology and a member of the Washington University Cancer Center. Her research interests focus on screening for early detection of and genetic susceptibility to cancer.

MATTHEW W. KREUTER, PhD, MPH, is Director of the Health Communication Research Laboratory, and assistant professor of Behavioral Science/Health Education, Department of Community Health. Recent publications focus on tailored health communication and disease prevention in primary-care settings.

SARAH KOBRIN, MPH, is a doctoral student and research associate at the Health Communications Research Laboratory. Presently she is conducting doctoral research on measurement of perceived risk of breast cancer.

VICTOR J. STRECHER, PhD, MPH, is Director of Cancer Prevention and Control at the University of Michigan Comprehensive Cancer Center. His research interests focus on use of interactive communication technologies for health education and health behavior change.

aCKnowledgements. This research was supported by Public Health Service Grant 1 R29 HL41886 from the National Heart Lung and Blood Institute to Dr Strecher and grants from the Lineberger Comprehensive Cancer Center and the William Randolf Hearst Foundation to Dr Kreuter.

ADDRESS. Correspondence should be directed to: CELETTE SUGG SKINNER, PhD, Mallinckrodt Institute of Radiology, Washington University School of Medicine, 510 South Kingshighway Boulevard, St Louis, Missouri 63110, USA.
Journal of Health Psychology Copyright (C) 1998 SAGE Publications London, Thousand Oaks and New Delhi, [1359-1053(199804)3:2]

Vol 3(2) 181-193; 003381

\section{Abstract}

Perceived risk can influence health behaviors. Studies using various populations and breast cancer risk bias assessment methods have identified both risk over- and underestimation. Among 1803 women in primary care settings, 47 percent were at average epidemiologic risk (Gailcalculated relative risk \pm 50 percent of age-adjusted population average) and 55 percent perceived themselves to be at average risk (compared to same-age others) but there were mismatches or 'biases': 31 percent underestimated personal risk; 26 percent overestimated. Multiple logistic regression revealed that smokers were more likely to overestimate risk. Overestimation decreased with more education. Mammography use did not independently predict perception bias but, among never-screened women aged over 40 years, those contemplating mammograms were most likely to overestimate risk; precontemplators were most likely to underestimate. Implications for research and intervention are discussed.

\section{Keywords}

bias, breast cancer, perception, risk 
THE CONCEPT OF perceived risk figures prominently into models explaining and predicting health behavior (Cummings, Becker, \& Maile, 1980; Fishbein \& Ajzen, 1975; Weinstein, 1988). Not until people perceive personal risk from a health threat do they have reason to consider modifying behaviors to reduce risk (Janz \& Becker, 1984; Strecher \& Rosenstock, 1997). Therefore, traditional health promotion interventions have sought to increase perceived risk. Organizations such as the American Cancer Society have sought to publicize the average woman's lifetime risk of developing breast cancer, presumably based on the idea that increased risk perception may translate into increased participation in breast cancer screening. However, we know very little about how well people understand risk information (Evans, Burnell, Hopwood, \& Howell, 1993; Polednak, Lane, \& Burg, 1991) and even less about how individuals' perceived risks compare with their actual risks.

Until recently, the conventional wisdom was to assume a widespread underestimation of personal risk. Weinstein $(1982,1988)$ identified this tendency toward 'optimistic bias', which may result from not understanding probabilistic risk information (Slovic, 1987), discounting or not even considering risk factor information in order to feel more comfortable (Abelson, 1983; Festinger, 1957; Weinstein, 1982), or from psychological mechanisms (e.g. heuristics) used to process complex risk information (Tversky \& Kahneman, 1991). Numerous studies have identified optimistic biases for a variety of health threats (Avis, Smith, \& McKinlay, 1989; Becker \& LeVine, 1987; Kreuter \& Strecher, 1995; Kulik \& Mahler, 1987; Lee, 1989; Strecher, Kreuter, \& Kobrin, 1995; Weinstein, 1980, 1982, 1983, 1984); most employing Weinstein's methodology (Weinstein, 1982, 1983, 1984) of operationally defining optimistic bias as a collective risk perception of all subjects relative to a similar index population. In response to the statement 'Compared to [similar others], my chances of developing [health problems] are:', subjects choose one of seven responses ranging from 'much below average' (assigned a value of $-3)$ to 'much above average' $(+3)$. Group responses are considered, with a negative group mean indicating optimistic bias (e.g. the average subject in the sample perceives his or her risk to be lower than similar others in the index population). Researchers using this methodology (Kulik \& Mahler, 1987; Lee, 1989; Weinstein, 1980, 1982) have concluded that women generally underestimate their breast cancer risks. For instance, Aiken and colleagues (Aiken, Fenaughty, West, Johnson, \& Luckett, 1995 ) found that only 16 percent of women aged 37 to 77 years thought that their breast cancer risk was higher than other women their age, while nearly 50 percent thought that their risk was below average. However, even among a sample with a collective optimistic bias, individual subjects vary in their personal risk factors, so that some may have accurate risk perceptions (e.g. they may be realists) and others may overestimate risk (e.g. hold pessimistic biases).

For intervention planning, it would be helpful to identify who is likely to over- or underestimate breast cancer risk and to understand the association of risk bias with participation in screening. Unfortunately, studies of breast cancer risk bias are inconsistent in questions asked, methods used, populations studied, and findings. Some have shown widespread risk overestimation. Black and colleagues (Black, Nease, \& Tosteson, 1995) found that women aged 40 to 50 years dramatically overestimate the risk of 'women exactly like you' dying of breast cancer in the next 10 years. Similarly, among women aged 35 years or older who had a first-degree relative with breast cancer, Lerman et al. (1991) found that about two-thirds extremely overestimated their risks, a quarter overestimated (although not extremely), fewer than 10 percent were accurate, and only 2 percent underestimated risk. In contrast, Evans et al. (1993) found that only 29 percent overestimated their risk by more than 50 percent, 23 percent underestimated by the same amount, and 44 percent of their study population (women aged 25 to 70 years with at least one family member with breast cancer) were able to come within 50 percent of a clinician's estimate. Lipkus, Rimer, and Strigo (1996) found that women with higher Gail-calculated risk also felt greater subjective risk, but the strength of this relationship was modest (correlation of 0.21).

This report describes the extent, direction and correlates of risk bias in a cross-sectional sample of 1803 female patients of community-based family practitioners, a substantially larger and 
more generalizable sample than those in most previous studies on breast cancer risk perceptions. We discuss possible implications of the findings for intervention and practice.

\section{Methods}

\section{Sample}

The sample and data collection methodology have been described previously (Kreuter, Strecher, Harris, Kobrin, \& Skinner, 1995). Adult patients from 12 community-based family practitioner groups in North Carolina completed a self-administered questionnaire assessing eight health-related behaviors and three screening practices including mammography. The questionnaire also assessed several theoretical constructs associated with screening (e.g. stage of adoption, perceived barriers, perceived risk) and included risk assessment questions derived from the Healthier People health risk appraisal (HRA). Trained graduate research assistants approached patients in physicians' waiting rooms and explained the study, determined age eligibility (18 to 75 years), and asked eligible patients to complete the questionnaire while waiting to see their health care provider.

Participation rates ranged from 65 percent to 95 percent across the 12 practices. Of 3772 eligible patients, 2971 (79 percent) agreed to participate. Refusers were more likely to be male andby research assistants' estimates-older than participants. Of the 2971 patients who completed the questionnaire, 5 percent $(n=153)$ were dropped from analysis due to incomplete data. Sixtyfour percent of the remaining 2818 respondents were female. Data from these 1803 women participants were used for this study.

\section{Risk measurements}

Perceived breast cancer risk was measured by asking women, 'Compared to others your same age and sex, how would you rate your risk of getting breast cancer within the next 10 years?' Five response categories ranged from 'a lot lower than average' to 'a lot higher than average'. Because few women classified their breast cancer risk as much higher or much lower than average, we collapsed risk perception responses into 'lower than average', 'average', and 'higher than average' when conducting bivariate and multivariate analyses.
Actual risk was computed using risk estimation algorithms from the Gail model (Gail \& Benichou, 1992; Gail et al. 1989), an unconditional logistic regression with relative associations of risk factors to determine a woman's probability of developing breast cancer. Developed at the National Cancer Institute by Mitchell Gail and Jacques Benichou, the Gail model is used to estimate a woman's absolute risk for developing breast cancer in the next 10 years. The model is based on five currently accepted risk factors for breast cancer: (1) age; (2) family history of breast cancer; (3) age at first menstrual period; (4) age at which a woman gives birth to her first child; and (5) the number of breast biopsies a woman has had. Risk increases as age increases, family history increases, age at first menstrual period decreases, age at first live birth increases, and number of breast biopsies increases. Our questionnaire assessed these five factors, with one limitation: we could code for whether a woman had had a previous breast biopsy $(0$ or $\geq 1)$ but could not distinguish between one versus more than one biopsy (the full Gail model coding of 0,1 , or $\geq 2$ ). Among the sample $(n=1803)$, there were relatively few missing values for the five Gail items: age at menarche was missing for 43 subjects; family history missing for 138 ; age at parity missing for 37 ; and biopsy information missing for 22 subjects. For these missing single risk factor values, we imputed conservative values equal to a relative risk of zero (Gail \& Benichou, 1992).

Because the Gail model calculates individual relative risk by comparing a risk derived from a regression equation (considering age, family history, menarche, biopsies, and parity) to a same-age woman who has no risk factors, 'average' risk is not 1.0 as it would be in a true relative risk. Instead, 1.0 is the lowest possible risk derived from the formula (i.e. no risk factors). To determine a true age-specific relative risk for each woman in the sample, we compared her risk to the average risk for a woman of the same age. These average, or 'composite risk' estimates were derived from the study population on which the Gail model was based (Gail \& Benichou, 1992). We thus obtained an age-adjusted relative risk. However, classification of women into risk bias categories necessitated an extension of relative risk into an average range rather than a discrete value. We 


\begin{tabular}{|l|l|l|l|}
\hline & $\begin{array}{l}\text { Perceived } \\
\text { higher than average }\end{array}$ & Perceived & Perceived \\
lower than average \\
\hline Actual & Realistic & Optimistic & Optimistic \\
\hline Actual & Pessimistic & Realistic & Optimistic \\
average & Pessimistic & Pessimistic & Realistic \\
\hline $\begin{array}{l}\text { Actual } \\
\text { lower than average }\end{array}$ & & & \\
\hline
\end{tabular}

Figure 1. Breast cancer risk bias classification.

used a conservative interval defining average relative risk as those scores from 50 percent higher or lower than a true average, inclusive. Forty-seven percent of subjects had a relative breast cancer incidence risk in this range; 33 percent were in the lower-than-average risk category and 20 percent were in the higher-thanaverage risk category.

Risk perception bias was calculated using techniques similar to those reported by Avis and colleagues in their investigation of risk perception for heart disease (Avis, 1989). We compared actual epidemiologic risk and perceived risk to classify women as having optimistically biased, realistic, or pessimistically biased risk perceptions. As Figure 1 illustrates, a woman was categorized as having an optimistic bias if her perceived risk of developing breast cancer was lower than her actual risk, as having a pessimistic bias if her perceived risk was higher than her actual risk, and as having a realistic perception if actual and perceived risks 'matched'. In this article, women may be referred to as 'optimists' (who underestimate risk), 'pessimists' (who overestimate risk), or 'realists' (without risk perception biases).

\section{Analyses}

Bivariate associations between demographic variables and the three-level perceived risk bias variable (optimistic, realistic, or pessimistic) were assessed using chi-square tests. Chi-square and $p$ values are reported. Further to describe risk perception biases, we regressed dichot- omous optimism and pessimism variables (optimistic/not optimistic; pessimistic/not pessimistic) on four demographic variables using multiple logistic regression. Descriptive variables included dichotomous measures for race (white/non-white), marital status (married/not married), smoking status (current smoker/nonsmoker) and a continuously scaled variable for years of education completed. These analyses report an odds ratio (OR) statistic estimating the magnitude of association between these variables and the dichotomous risk bias variable by comparing the odds of the risk bias occurring in different levels of the demographic variables (Hennekens \& Buring, 1987). An OR of 1.0 indicates no association, $\mathrm{OR}>1.0$ indicates a positive association between the variables, and OR $<1.0$ indicates a negative association.

\section{Results}

\section{Sample characteristics}

Characteristics of the sample are indicated in Table 1. Participants were mostly married, white, and had at least 12 years of education. Mean age was 40 years. Fifteen per cent reported a family history of breast cancer.

More than half of all women in the sample (58 percent) had had at least one mammogram. A large majority of women aged 40 years and older ( 87 percent) had had at least one mammogram and 55 percent of the women over 50 had had screening mammograms within the previous two years. Nearly half (46 percent) of women in 
Table 1. Sample characteristics

\begin{tabular}{lr}
\hline & $\%$ \\
\hline Marital status & \\
$\quad$ Married & 64 \\
Single & 17 \\
$\quad$ Previously married & 18 \\
Race & \\
White & 82 \\
African-American & 16 \\
Other & 2 \\
Education (years) & \\
$<12$ & 14 \\
12 & 37 \\
$13-16$ & 24 \\
$>16$ & 24 \\
Age (years) & \\
$<30$ & 24 \\
$30-39$ & 31 \\
$40-49$ & 23 \\
$50-59$ & 12 \\
$\geq 60$ & 10 \\
Family history of breast cancer & 15 \\
\hline
\end{tabular}

their thirties, for whom routine mammograms are not generally recommended, had had at least one mammogram.

\section{Perceived risk}

Distributions of perceived breast cancer risk compared to other women the respondent's same age are displayed in Table 2.

Perceived risk differed significantly by race, education level, and smoking status, but not by marital status or age. Forty-four percent of nonwhite women, compared with 30 percent of white women, perceived their risk as lower than average. Risk perception varied by education level, with women who had fewer years of education more likely to view themselves as having average breast cancer risk. Perceived risk

Table 2. Perceived risk

\begin{tabular}{lr}
\hline & $\%$ \\
\hline Much higher than average & 1 \\
Higher than average & 11 \\
Average & 55 \\
Lower than average & 24 \\
Much lower than average & 9 \\
\hline
\end{tabular}

also varied significantly by smoking status: only 22 percent of current smokers saw themselves as being at lower than average risk for breast cancer, compared with 32 percent of former smokers and 37 percent of women who had never smoked.

Higher perceived breast cancer risk was significantly related to having ever had a mammogram. Among the sample as a whole, 70 percent of those who saw themselves as being at higher than average risk for breast cancer had had a mammogram, compared with 57 percent of those who saw themselves at average risk and 53 percent who thought their risk was lower than average. The relationship between perceived susceptibility and having had at least one mammogram persisted after controlling for age. Among women aged 40 years and over, for whom screening intervals were recommended by national organizations at the time the study was conducted (American Cancer Society, 1983), the lower the women's perceived susceptibility to breast cancer, the less likely they were to have received a mammogram within the recommended time period.

Women reporting a family history of breast cancer were more likely than those without a breast cancer family history to view themselves as being at higher than average risk: 37 percent vs 8 percent, respectively. However, almost half (47 percent) of all women with a family history of breast cancer viewed their risk as average and 16 percent felt that their breast cancer risk was even lower than average compared with other women their age.

Multivariate analysis entering age and family history into the same ordinal logistic regression model likewise revealed a significant effect of family history on perceived risk for breast cancer but no significant effect of age on perceived risk. Neither was there a significant age by family history interaction effect on perceived risk.

\section{Risk bias}

Biases in risk perceptions were assessed by comparing actual and perceived risks. Although roughly half of the women (47 percent) were at average actual risk (Gail-calculated relative risk \pm 50 percent of age-adjusted population average risk) and just over half (55 percent) perceived themselves as being at average risk, there were 
numerous mismatches or biases in risk perceptions. When individual subjects' actual and perceived risks were compared, 31 percent of the sample underestimated and 26 percent overestimated their breast cancer risk. Findings from bivariate analyses revealed that risk perception did not vary significantly by race or marital status but bias was related to education level, smoking status, and whether the woman had ever had a mammogram.

Significant variations in risk bias by education level were as follows (see Table 3). Likelihood of holding a pessimistic bias was higher among women with less education. Those in the highest and lowest education groups were most likely to be optimists. Least educated women were least likely to be realists. Most educated were least likely to be pessimists. Compared to those with more or fewer years of schooling, women with 12 years of education were more likely to be pessimists. This association between education and risk bias remained after controlling for age and there was not a significant age by education interaction (looking at the outcome of risk bias category).

Risk bias also varied significantly by smoking status (Table 3). Current smokers were less likely than non-smokers to hold an optimistic bias and more likely to overestimate risk; percentages of smokers and non-smokers with realistic risk perceptions did not differ appreciably.

Table 3. Bivariate relationships of risk bias and demographic variables

\begin{tabular}{|c|c|c|c|c|c|}
\hline & Optimistic (\%) & Realistic (\%) & Pessimistic (\%) & Statistic & $p$ value \\
\hline \multicolumn{6}{|l|}{ Race } \\
\hline White & 29 & 43 & 26 & & \\
\hline Non-white & 35 & 40 & 25 & $\chi^{2}=3.20$ & .2005 \\
\hline \multicolumn{6}{|l|}{ Marital status } \\
\hline Married & 29 & 43 & 27 & & \\
\hline Not married & 34 & 42 & 24 & $\chi^{2}=4.56$ & .1022 \\
\hline \multicolumn{6}{|l|}{ Age (years) } \\
\hline$<30$ & 24 & 42 & 34 & & \\
\hline $30-39$ & 22 & 44 & 34 & & \\
\hline $40-49$ & 28 & 47 & 25 & & \\
\hline$\geq 50$ & 53 & 39 & 8 & $\chi^{2}=164.362$ & $<.0001$ \\
\hline \multicolumn{6}{|l|}{ Education (years) } \\
\hline$<12$ & 37 & 36 & 27 & & \\
\hline 12 & 28 & 42 & 40 & & \\
\hline $13-15$ & 26 & 48 & 26 & & \\
\hline$\geq 16$ & 37 & 45 & 18 & $\chi^{2}=37.088$ & $<.0001$ \\
\hline Mean education & 13.1 years & 13.3 years & 12.8 years & $F=6.15$ & .0022 \\
\hline \multicolumn{6}{|l|}{ Smoking status } \\
\hline Smoker & 21 & 40 & 39 & & \\
\hline Non-smoker & 33 & 44 & 22 & $\chi^{2}=45.38$ & $<.0001$ \\
\hline \multicolumn{6}{|c|}{ a Ever had mammogram? } \\
\hline Yes & 40 & 44 & 16 & & \\
\hline No & 37 & 41 & 22 & $\chi^{2}=34.655$ & $<.0001$ \\
\hline \multicolumn{6}{|c|}{ a Due for mammogram? } \\
\hline Yes & 43 & 42 & 15 & & \\
\hline No & 38 & 44 & 17 & $\chi^{2}=1.83$ & .4013 \\
\hline \multicolumn{6}{|l|}{ Family history? } \\
\hline Yes & 63 & 37 & 0 & & \\
\hline No & 26 & 44 & 30 & $\chi^{2}=201.68$ & $<.0001$ \\
\hline
\end{tabular}

${ }^{\text {a }}$ Women aged $\geq 40$ years only. 
Table 4. Multiple logistic regression findings for dichotomized risk bias variables

\begin{tabular}{lcccccc}
\hline Term & Estimate & $S E$ & $\chi^{2}$ & p value & OR & 95\% CI \\
\hline Parameter estimates for dichotomized & pessimistic & bias variable & & & \\
Smoking & -0.371 & 0.06 & 33.92 & 0.00 & 0.69 & $0.61-0.78$ \\
Marital status & 0.104 & 0.00 & 2.87 & 0.09 & 1.11 & $0.98-1.25$ \\
Race & 0.036 & 0.06 & 0.20 & 0.66 & 1.04 & $0.89-1.21$ \\
Education & 0.063 & 0.03 & 5.67 & 0.02 & 1.07 & $1.01-1.12$ \\
Parameter estimates for & dichotomized & optimistic bias variable & & & \\
Smoking & 0.306 & 0.07 & 18.8 & 0.000 & 1.36 & $1.18-1.56$ \\
Marital status & -0.114 & 0.06 & 4.00 & 0.045 & 0.89 & $0.80-1.00$ \\
Race & -0.089 & 0.07 & 1.47 & 0.225 & 0.91 & $0.79-1.06$ \\
Education & 0.002 & 0.03 & 0.01 & 0.929 & 1.00 & $0.95-1.05$ \\
\hline
\end{tabular}

For the subset of 754 women aged forty and over, for whom routine mammography screening was recommended at the time of interview, we assessed whether risk biases varied by mammography status. Risk biases did not vary significantly by whether women were currently compliant with screening guidelines or were due for a mammogram but there was significant variation by whether they had ever had a mammogram (Table 3). Women who had had at least one mammogram were more likely to be optimists or realists and less likely to be pessimists than were women who had never had a mammogram. We conducted similar analyses among subsets of women in their forties and their thirties, but found no significant associations between risk perception bias and mammography use.

As is shown in Table 3, women aged 50 and over were most likely to be optimistic and least likely to be pessimistic; women younger than 40 were most likely to hold pessimistic biases. Women with family histories of breast cancer were more likely to hold optimistic biases and were less likely to hold pessimistic biases or have realistic risk perceptions than were women without family histories of breast cancer.

To identify independent predictors after controlling for other variables, we used multiple nominal logistic regression to examine associations among these demographic variables and women's likelihood of holding optimistic or pessimistic biases. We regressed dichotomous risk bias variables (optimistic/not optimistic and pessimistic/not pessimistic) on race, marital status, education, and smoking status. Results including parameter estimates, standard errors, chi-square values, $p$ values, standard errors, odds ratios, and 95 percent confidence intervals for each of these variables are presented in Table 4.

The multiple nominal logistic regression results confirmed findings from bivariate analyses. Optimistic bias for breast cancer risk was less likely among smokers (Table 4). Pessimistic bias was more likely among smokers and the probability of holding pessimistic biases decreased with more years of education. It should be noted that, although the likelihood of being a current smoker was significantly inversely associated with increasing years of education, both education and smoking status maintained significant independent effects in the regression model.

For the subset of 754 women aged 40 and over, we repeated the above multiple logistic regression analyses, including the mammography variables. In these subset analyses, marital status and smoking status were significantly associated with pessimistic bias and optimistic bias (Table 4). However, neither whether women had ever had a mammogram nor whether they had had one within the recommended time frame were significantly independently correlated with risk perception bias.

Identifying demographic correlates of breast cancer risk perception biases can help us understand who is most likely to have optimistic and pessimistic biases, but tells us little about how those perceptions influence, and are influenced by, women's breast cancer screening practices and intentions. It is possible that experience with prior mammography-especially an abnormal mammogram and subsequent work-up-might 
be associated with risk perception biases. To explore this relationship, we examined risk perception biases among women who varied in mammography history, breast surgery, surgery outcome, and stage of considering mammography. Based on the Transtheoretical model and our previous methodology (Prochaska \& DiClemente, 1983; Skinner, Strecher, \& Hospers, 1994), woman who had not had mammograms were classified as contemplators (seriously thinking about having a mammogram within the following six months) or precontemplators (not thinking about having a mammogram).

Among those aged at least 40 and thus old enough to be screened, the highest proportion of optimistic bias (51 percent) and lowest proportion of pessimistic bias (11 percent) were found in women who had had a mammogram, had breast surgery, and had a negative surgery finding (see Figure 2). Among those who had had mammograms but had never had breast surgery, comparatively more were realistic (45 percent) or had pessimistic biases (17 percent) and fewer held optimistic biases (38 percent). Women who had never had a mammogram but were seriously thinking about it (i.e. contemplators) were least likely to hold optimistic biases (30 percent) and most likely, of any group, to be pessimistically biased (35 percent). Women who had never received a mammogram and were not thinking about getting one (i.e. precontemplators) mostly had optimistic biases (41 percent) or were realistic (43 percent): only 16 percent held pessimistic biases.

\section{Discussion}

Risk perceptions have received a fair amount of attention in recent years. Results from previous studies have varied, depending on sample makeup and method of assessing risk bias. Most studies using Weinstein's methodology of operationalizing risk bias as a collective risk perception of all subjects relative to a similar index population (Weinstein, 1980, 1982, 1983, 1984) have identified general tendencies toward optimistically biased risk perceptions. Other studies, asking women about their own risk or the risk of women 'exactly like' themselves in probabilistic terms within a designated time frame, have identified gross risk overestimation.

Our study employed elements from both methodologies. We asked women to compare their risk with that of others the same age, as did Aiken and colleagues (1995), while using the Gail formula to estimate individuals' actual risks, as did Black et al. (1995), Lerman et al. (1995) and Lipkus, Rimer, and Strigo (1996).

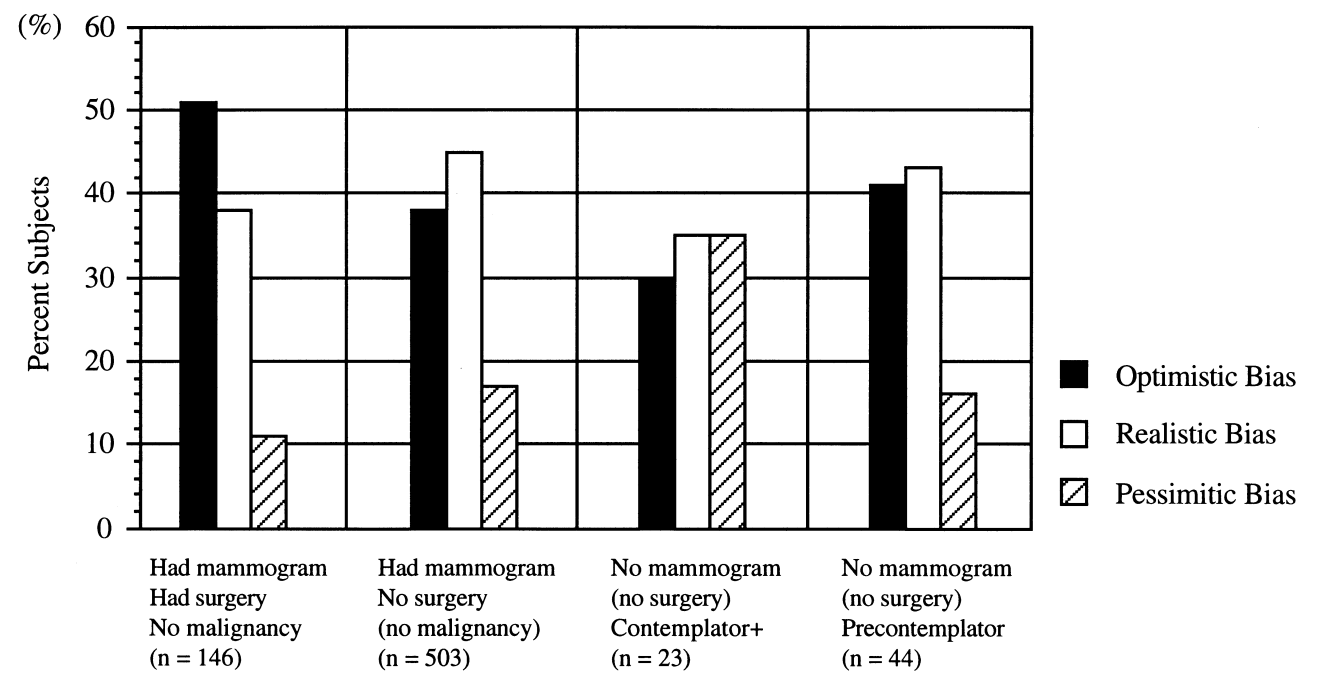

Figure 2. Breast cancer risk perception biases by mammography history, breast surgery, surgery outcome, and stage of considering mammography. 
Using a behavioral-attitudinal questionnaire and a measure of individual risk factors, we identified and compared perceived and actual breast cancer risks among a sample of women and determined whether individual subjects had optimistically biased, realistic, or pessimistically biased perceptions of their personal risk. Few investigators have been able to compare perceived risk relative to others the woman's same age and her actual epidemiologic risk. This study of 1803 women is one of the first to indicate the prevalence of optimistic and pessimistic breast cancer risk biases among a sizable sample of women seen in community-based primary practice settings and to identify correlates of risk biases. Although many of our analyses have focused on the 57 percent of the sample that displayed risk perception biases, readers should note that a sizable percentage of the sample (43 percent) had realistic risk perceptions.

Our findings make an important methodological point that is often ignored in the risk bias literature: in a relatively healthy sample, it is possible for a majority to rate their risk below average without being biased. Because a much larger percentage rated themselves at below average ( 33 percent) than above average (12 percent) risk, the sample would have appeared to be optimistically biased, somewhat like that of Aiken et al. (1995), had the study been conducted with the traditional method of operationally defining bias as a collective risk perception of all subjects relative to a similar index population. However, by assessing risk bias at the individual rather than the collective level, the study showed that the majority of women did not have an optimistic bias: only 31 percent underestimated their risk. Further, results among this large sample from community-based primary care settings reveal less risk overestimation than previous studies of perceived and actual risk among breast cancer patients' relatives.

Bivariate analyses showed that both mostand least-educated women held risk biases. This finding has intervention implications: rather than assuming that realistic risk perception comes with education, we should emphasize breast cancer susceptibility for women at all education levels.

Findings also indicated that cigarette smoking was associated with pessimistic bias. This could indicate a belief that smoking is a risk factor for breast cancer. Alternatively, smokers may rate their breast cancer risk as higher, not because they smoke but because they also engage in other behaviors they perceive as affecting their risk (i.e. high-fat diet or lack of exercise). Investigators have previously found that current smokers are less likely than non-smokers to undergo regular mammography (McBride, Curry, Taplin, Anderman, \& Grothaus, 1993), but this was not the case in our sample. Among women aged 40 years and over, there was no significant variation in mammography use between smokers and non-smokers. Intervention implications for the finding that smokers overestimate their breast cancer risks must be considered with care. It is doubtful whether health educators would relish the opportunity of telling smokers that their tobacco use probably does not increase their breast cancer risk. Rather, intervention messages might be designed to redirect heightened risk perceptions to conditions such as heart disease and lung and cervical cancers, for which smoking is a well-established risk factor (Bartecchi, MacKenzie, \& Schrier, 1994). Further, women smokers may need to be reminded that lung cancer is the number one cause of cancer deaths among US women (Bartecchi, MacKenzie, \& Schrier, 1994) and that smoking cessation is a crucial cancer risk reduction measure.

An important limitation in considering the relationship of age and family history on risk bias is that both age and family history are factored in to a woman's Gail-calculated actual risk and thus affect risk-bias calculation. For instance, having a family history of breast cancer increases actual risk to such a degree that it becomes statistically unlikely for a woman with family history to overestimate her risk enough to be classified in the pessimistic bias category. Women with higher actual risk have more 'room' to be optimistic than women with lower actual risk because of the floor and ceiling effects of these measures. To some extent, findings might be artifacts of the measure. Although our finding that women with a family history were more likely to have optimistic biases and less likely to have pessimistic risk biases must be interpreted with caution, a look at optimistic bias (or risk underestimation) 
seemed warranted in this sample because we found such a large group of women with family history of breast cancer who viewed themselves as being at average risk (47 percent) or even below average (16 percent) risk, compared with other women their same age. However, because age and family history are both included in the Gail model risk score and are thus factored into pessimistic and optimistic risk bias algorithms, we did not include age or family history in multiple regression analyses.

It is possible that women with a family history were considering other factors when assessing their own risk. For example, if they knew that their own risk would be affected more by breast cancer in a premenopausal relative, they might have discounted the extent to which an older relative's cancer affected their risk. However, preliminary investigation of firstdegree relatives' risk perceptions has suggested that few women realize the difference in risk, depending on their relative's age at diagnosis (Ryan \& Skinner, 1995). Another possibility is that women who knew they were at heightened familial risk might have made lifestyle changes that they perceived would lower their risk. Whether such changes actually lower risk in first-degree relatives has not yet been determined. Our findings indicate that there may be a substantial number of women with family histories of breast cancer who do not understand that their personal breast cancer risk is affected by the presence of breast cancer in a close family member. More research is needed to explore this issue more fully.

These associations between family history and optimistic bias may seem incongruent with previous reports that people are most likely to be optimistically biased in regard to risks that are most controllable and more realistic about less controllable genetic and environmentally determined risks (Weinstein, 1982). Although genetic risk is uncontrollable, the degree to which women with a family history view their breast cancer risk as either genetic or controllable is unclear. Investigators studying lay perceptions of breast cancer risk have identified numerous factors perceived by various groups (Chavez, Hubbell, McMullin, Martinez, \& Shiraz, 1995; Payne, 1990; Ryan \& Skinner, 1995). It may be that women with a family history do not consider the possibility of having inherited a genetic predisposition for breast cancer or they may believe that they have lowered their inherited risk by making lifestyle changes (e.g. in diet or exercise).

Because some have argued that optimistic biases may discourage desirable health behaviors (Burger \& Burns, 1988), we expected that risk bias might affect mammography status. In bivariate analyses among women aged 40 and over, there was a significant association between risk bias and having had at least one mammogram (e.g. those who had had at least one were less likely to be pessimistic than women who had never had a mammogram), but mammography use did not emerge as a significant independent predictor in multivariate analyses. Readers should note that the numbers of women in the precontemplation and contemplation stages were small and the results should be cautiously interpreted. However, it is possible that, as Taylor and Brown (1988) have suggested, optimistic biases may not inhibit-and may even promote-health-protecting behaviors. The high mammography rates among women from the 12 prevention-oriented primary practice groups participating in the study may have limited our ability to detect between-group differences and these associations should, therefore, be explored in a sample of women with a more normal distribution of mammography use.

Since mammography screening has been associated with perceived susceptibility for breast cancer (Champion, 1992; Glockner, Holden, Hilton, \& Norcross, 1992; Kruse \& Phillips, 1987; Lerman, Rimer, Trock et al., 1990; Lerman et al., 1991; Rutledge, Hartmann, Kinman, \& Winfield, 1988; Zapka, Stoddard, Constanza, \& Maul, 1989), it seems that interventions should continue to emphasize breast cancer risk, especially among women with family histories. Steps should be taken to ensure the dissemination of this message to women of all ages and education levels.

A person's perception of self-risk can be influenced by many factors, including past experience, defensive avoidance, denial, egocentric tendencies (thinking about one's self outside the context of others, i.e. ignoring the precautions others are taking), and cognitive errors (Weinstein, 1980, 1982, 1984). Our paper focuses not on these types of factors, but rather on correlates of risk perception biases. However, some of those correlates (i.e. mammography 
history, surgery outcome, and stage of considering mammography) are at least capable of influencing-not just co-varying with-perceived risk. In addition to identifying demographic correlates of breast cancer risk perception biases, we examined risk perception biases among women who varied in mammography history, breast surgery, surgery outcome, and consideration of future mammography. We looked at the relationship between biases and combinations of these factors to see if there appear to be patterns of possible influence. Our data suggest that there might be. Taken together, the findings suggest that, among women who have not had a mammogram, concern about breast cancer (i.e. perceived risk) is associated with consideration of mammography, and is greatest among women seriously thinking about being screened. More interestingly, these data suggest that women receiving mammography, especially when it is followed by breast surgery finding no cancer, are more likely to have optimistic biases among their future breast cancer risk. These findings must be interpreted with caution because (1) previous biopsy affects Gail score and may affect risk perception and (2) the analysis was conducted on a small subset of the sample. Although our sample size is small for some of these categories (e.g. those who have had previous biopsies), we believe that our data point to an issue that researchers should explore in future studies of risk perception and mammography use: whether a normal mammogram or breast surgery finding no cancer may be interpreted by some women as a clean bill of health or evidence of low breast cancer risk for the future. If this is the case, and if these biases lead women to be less vigilant in complying with screening recommendation in the future, it may be necessary to reconsider educational strategies or messages provided to women during mammography.

Comparisons of individuals' perceived and actual epidemiologic risks should be continued. Research should determine whether the proportion with realistic risk perceptions is higher or lower for other health problems, using several different measures of accuracy from the one used here (which was necessarily conservative and may have overestimated the number of accurate or pessimistic responses).

Future research should also explore these issues among different samples. Ours was a large convenience sample of women seen by 12 family practice groups in the Piedmont section of North Carolina. The degree to which findings are generalizable to women in other geographical and sociological settings remains to be determined. For instance, risk perceptions of these patients of family practitioners in the southeastern United States may differ from risk perceptions of women in urban minority communities or from women who do not receive routine primary care.

A final limitation stems from our inability to perfectly measure actual risk. For estimating objective breast cancer incidence risk, it is common to choose between the Gail (Gail et al., 1989; Gail \& Benichou, 1992) and Claus (Claus, Risch, \& Thompson, 1994) models. Each has limitations. Gail considers personal risk factors such as age at menarche and parity. Claus concentrates on familial risk. Because the degree to which personal and/or lifestyle risk factors add significantly to inherited risk remains unclear, no model combining the contribution of all known risk factors has been verified. Thus, to date, any comparison of perceived and actual breast cancer risk-or any other cancer risk-is inherently limited. Further, there is a question of what to do when a value factored into the Gail model is missing for a particular subject. Our sample included few missing data. However, when we did encounter missing data, we imputed conservative values, so as not to assign a woman a risk factor she might not actually have. It is possible that there were subjects whose true values for the missing items would have conferred a higher risk to the person. Thus, our conservative approach may have biased towards pessimism for the few women with missing data, in that their actual risk might have been rated as lower than it truly was.

Realistic risk perceptions may always be desirable, but understanding personal and familial breast cancer risk may become both increasingly important and complicated as genetic risk evaluation (Biesecker et al., 1993; King, Rowell, \& Love, 1993) and expanded prevention options (Nayfield, Karp, Ford, Dorr, \& Karmer, 1991; Temple, Lindsay, Magi, \& Urbanski, 1991) become more widely available. Whereas breast cancer screening interventions have traditionally been designed to increase all women's percep- 
tions of breast cancer risk (Skinner, Strecher, \& Hospers, 1994), these new health care options may necessitate a more careful communication of realistic personal risk. Risk biases-both optimistic and pessimistic-may have more widespread implications in the future.

\section{References}

Abelson, R. (1983). Whatever became of consistency theory? Personality and Social Psychology

Bulletin, 9, 37-54.

Aiken, L. S., Fenaughty, A. M., West, S. G., Johnson, J. J., \& Luckett, T. L. (1995). Perceived determinants of risk for breast cancer and the relations among objective risk, perceived risk and screening behavior over time. Women's Health: Research on Gender, Behavior and Policy, 1, 27-50.

American Cancer Society. (1983). Mammography guidelines. Background statement and update of cancer-related check up for breast cancer detection in asymptomatic women age 40-49. $C A-A$ Cancer Journal for Clinicians, 33, 255.

Avis, N. E., Smith, K. W., \& McKinlay, J. B. (1989). Accuracy of perceptions of heart attack risk: What influences perceptions and can they be changed? American Journal of Public Health, 79, 16081612.

Bartecchi, C. E., MacKenzie, T. D., \& Schrier, R. W. (1994). The human costs of tobacco use (First of two parts). New England Journal of Medicine, 30, 907-912.

Becker, D. M. \& LeVine, D. M. (1987). Risk perception, knowledge, and lifestyles in siblings of people with premature coronary disease. American Journal of Preventive Medicine, 1, 45-50.

Biesecker, B. B., Moehnke, M., Calzone, K., Market, D. S., Barber, J. E., Collins, F. S., \& Weber, B. L. (1993). Genetic counseling for families with inherited susceptibility to breast and ovarian cancer. Journal of the American Medical Association, 269, 1970-1974.

Black, W. C., Nease, R. F., \& Tosteson, A. N. A. (1995). Perceptions of breast cancer risk and screening effectiveness in women younger than 50 years of age. Journal of the National Cancer Institute, 87, 720-731.

Burger, J. M. \& Burns, L. (1988). The illusion of unique invulnerability and the use of effective contraception. Personality and Social Psychology Bulletin, 14, 264-270.

Champion, V. L. (1992). Compliance with guidelines for mammography screening. Cancer Detection and Prevention, 16(4), 253-258.

Chavez, L. R., Hubbell, F. A., McMullin, J. M.,
Martinez, R. G., \& Shiraz, S. I. (1995).

Understanding knowledge and attitudes about breast cancer. Archives of Family Medicine, 4, 145-152.

Claus, E. B., Risch, N., \& Thompson D. (1994). Autosomal dominant inheritance of early-onset breast cancer: Implications for risk prediction. Cancer, 73, 643-651.

Cummings, K. N., Becker, M. H., \& Maile, M. C. (1980). Bringing the models together: An empirical approach to combining variables used to explain health actions. Journal of Behavioral Medicine, 3, 123-145.

Evans, D. G. R., Burnell, L. D., Hopwood, P., \& Howell, A. (1993). Perception of risk in women with a family history of breast cancer. British Journal of Cancer, 67, 612-614.

Festinger, L. (1957). A theory of cognitive dissonance. Stanford: Stanford University Press.

Fishbein, M., \& Ajzen, I. (1975). Belief, attitude, intention, and behavior: an introduction to theory and research. Reading, MA: Addison-Wesley.

Gail, M. H., \& Benichou, J. (1992). Assessing the risk of breast cancer in individuals. In V. T. De Vita Jr., S. Hellman and S. A. Rosenberg (Eds.), Cancer prevention (pp. 1-15). Philadelphia: Lippincott.

Gail, M. H., Brinton, L. J., Byar, D. P., Corle, D. K., Green, S. B., Schairer, C., \& Mulvihill, J. J. (1989). Projecting individualized probabilities of developing breast cancer for white females who are being examined annually. Journal of the National Cancer Institute, 81, 1879-1886.

Glockner, S. M., Holden, M. G., Hilton, S. V. W., \& Norcross, W. A. (1992). Women's attitudes toward screening mammography. American Journal of Preventive Medicine, 8, 69-77.

Hennekens, C. H., \& Buring, J. E. (1987). Epidemiology in medicine (pp. 77-82). Boston, MA: Little, Brown.

Janz, N. K., \& Becker, M. H. (1984). The health belief model: A decade later. Health Education Quarterly, 11, 1-47.

King, M. C., Rowell, S., \& Love, S. M. (1993). Inherited breast and ovarian cancer: What are the risks? What are the choices? Journal of the American Medical Association, 269, 1975-1980.

Kreuter, M. W., \& Strecher, V. J. (1995). Changing inaccurate perceptions of health risk: Results from a randomized trial. Health Psychology, 14, 56-63.

Kreuter, M. W., Strecher, V. J., Harris, R., Kobrin, S. C., \& Skinner, C. S. (1995). Are patients of women physicians screened more aggressively? A prospective study of physician gender and screening. Journal of General Internal Medicine, 10, 119-125. 
Kruse, J., \& Phillips, C. M. (1987). Factors influencing women's decisions to undergo mammography. Obstetrics and Gynecology, 10, 744-748.

Kulik, J. A., \& Mahler, H. I. M. (1987). Health status, perceptions of risk and prevention interest for health and non-health problems. Health Psychology, 6, 15-27.

Lee, C. (1989). Perceptions of immunity to disease in adult smokers. Journal of Behavioral Medicine, 12, 267-277.

Lerman, C., Lustbader, E., Rimer, B., Daly, M., Miller, S., Sands, C., \& Balshem, A. (1995). Effects of individualized breast cancer risk counseling: A randomized trial. Journal of the National Cancer Institute, 87, 286-292.

Lerman, C., Rimer, B., Trock, B., Balshem, A., \& Engstrom, P. F. (1990). Factors associated with repeat adherence to breast cancer screening. Preventive Medicine, 19, 279-290.

Lerman, C., Trock, B., Rimer, B., Boyce, A., Jepson, C., \& Engstrom, P. F. (1991). Psychological and behavioral implications of abnormal mammograms. Annals of Internal Medicine, 114, 657-661.

Lipkus, I. M., Rimer, B. K., \& Strigo, T. S. (1996). Relationships among objective and subjective risk for breast cancer and mammography stages of change. Cancer Epidemiology Biomarkers \& Prevention, 5, 1005-1011.

McBride, C. M., Curry, S. H., Taplin, S., Anderman, C., \& Grothaus, L. (1993). Exploring environmental barriers to participation in mammography screening in an HMO. Cancer Epidemiology, Biomarkers \& Prevention, 2, 599-605.

National Cancer Institute. (1987). Working guidelines for early cancer detection. Bethesda, MD.

Nayfield, S. G., Karp, J. E., Ford, L. G., Dorr, F. A., \& Karmer, B. S. (1991). Potential role of tamoxifen in prevention of breast cancer. Journal of the National Cancer Institute, 83, 1450-1459.

Payne, S. (1990). Lay representations of breast cancer risk. Psychology and Health, 5, 1-11.

Polednak, A. P., Lane, D. S., \& Burg, M. A. (1991). Risk perception, family history, and use of breast cancer screening tests. Cancer Detection and Prevention, 15, 257-263.

Prochaska, J. O., \& DiClemente, C. C. (1983). Stages and processes of self-change of smoking: toward an integrative model of change. Journal of Consulting and Clinical Psychology, 51, 390-395.

Rutledge, D. N., Hartmann, W. H., Kinman, P. O., \& Winfield, A. C. (1988). Exploration of factors affecting mammography behaviors. Preventive Medicine, 17, 412-422.

Ryan, E. L., \& Skinner, C. S. (1995). Risk beliefs and interest in counseling: Focus group interviews among first-degree relatives of breast cancer patients. Presentation, Society of Behavioral Medicine, San Diego.

Skinner, C. S., Strecher, V. J., \& Hospers, H. (1994). Physician recommendations for mammography: Do tailored messages make a difference? American Journal of Public Health, 94, 43-49.

Strecher, V. J., \& Rosenstock, I. M. (1997). The Health Belief Model. In K. Glanz, F. Lewis \& B. K. Rimer (Eds.), Health Behavior and Health Education (pp. 41-59). San Francisco, CA: Jossey-Bass, Inc.

Strecher, V. J., Kreuter, M. W., \& Kobrin, S. C. (1995). Do cigarette smokers have unrealistic perceptions of their heart attack, cancer, and stroke risks? Journal of Behavioral Medicine, 18, 45-54.

Slovic, P. (1987). Perception of risk. Science, 236, 280-285.

Taylor, S. E., \& Brown, J. D. (1988). Illusion and well-being: A social psychological perspective on mental health. Psychological Bulletin, 103, 193-210.

Temple, W. J., Lindsay, R. L., Magi, E., \& Urbanski, S. J. (1991). Technical considerations for prophylactic mastectomy in patients at high risk for breast cancer. American Journal of Surgery, 161, 413-414.

Tversky, A., \& Kahneman, D. (1991). The framing of decisions and the psychology of choice. Science, 211, 453-458.

Weinstein, N. D. (1980). Unrealistic optimism about future life events. Journal of Personality and Social Psychology, 39, 806-820.

Weinstein, N. D. (1982). Unrealistic optimism about susceptibility to health problems. Journal of Behavioral Medicine, 5, 441-460.

Weinstein, N. D. (1983). Reducing unrealistic optimism about illness susceptibility. Health Psychology, 2, 11-20.

Weinstein, N. D. (1984). Why it won't happen to me: Perceptions of risk factors and illness susceptibility. Health Psychology, 3, 431-457.

Weinstein, N. D. (1988). The precaution adoption process. Health Psychology, 7, 355-386.

Zapka, J. G., Stoddard, A. M., Constanza, M. E., \& Maul, A. C. (1989). Breast cancer screening by mammography: Utilization and associated factors. American Journal of Public Health, 79, 14991502. 